\title{
Lectura musical vicaria. Lectura, comunidades interpretativas y discurso sonoro: el caso del Hei mihi Domine de Francisco Guerrero y su lectura en La Catedral de Bogotá*
}

Miguel Fonseca Martínez**

\section{Resumen}

El objetivo fundamental del presente artículo consiste en vincular el concepto de comunidades interpretativas a una posible hermenéutica de la música. De este modo, el lenguaje musical podría ser interpretado más fácilmente si se describe analógicamente con su raíz lingüística. De ello deviene que una descripción de las prácticas y comunidades interpretativas de la música estaría ampliamente emparentada con las prácticas y comunidades interpretativas de los lenguajes naturales. Esta tentación metodológica quiere razonar a través de un caso. Se pretende ver la plausibilidad de la hipótesis con un experimento. El experimento consiste en mostrar cómo se leyó la música de Francisco Guerrero en la Bogotá de la Colonia, y señalar si en este caso existen relaciones con el desarrollo de la lectura de lenguajes naturales o si, por el contrario,

* Artículo de investigación. DOI: http://dx.doi.org/10.15332/s0120-8462.2017.0117.07

** Investigador becario de Colciencias, actualmente adelanta el Doctorado en Filosofía. Magister en Filosofía Latinoamericana de la Universidad Santo Tomás. Ha sido docente de la Universidad de La Salle y de la Jorge Tadeo Lozano. miguelfonseca111@gmail.com 
hay discontinuidades. Se quiere ver si el concepto de comunidades interpretativas puede dar cuenta en cierta medida de la dialéctica entre obra musical y el sentido extratextual del lenguaje musical. Se intentará mostrar así que quizá la música no sea solamente un lenguaje sintáctico, sino que su semántica puede devenir de lo extramusical que se cuela en las prácticas de quien la escribe y quien la lee.

Palabras clave: comunidades interpretativas, discurso sonoro, lectura musical vicaria, Francisco Guerrero, lectores aficionados, lectores profesionales.

\title{
Vicarious musical reading. Reading, interpretive communities and sound discourse: the case of the Hei mihi Domine by Francisco Guerrero and his reading in The Cathedral of Bogotá
}

\author{
Miguel Fonseca Martínez
}

\section{Abstract}

The fundamental objective of this article is to associate the concept of interpretive communities to a possible hermeneutics of music. Thus, the musical language could be interpreted more easily if described analogically with its linguistic root. From this, a description of the practices and interpretive communities of music would be widely related to the practices and interpretive communities of natural languages. This methodological temptation wants to reason through a case. We intend to see the plausibility of the hypothesis with an experiment. The experiment consists of showing how the music of Francisco Guerrero was read in the Bogota of the Colony, and indicate if in this case there are relations with the development of the reading of natural languages or if, on the contrary, there are discontinuities. We want to see if the 
concept of interpretive communities can account in some extent to the dialectic between musical work and the extra-textual meaning of musical language. An attempt will be made to show that music may not be just a syntactic language, but that its semantics may come from the extra-musical that sneaks into the practices of the person who writes it and who reads it.

Keywords: interpretive communities, sound discourse, vicarious musical reading, Francisco Guerrero, amateur readers, professional readers.

\title{
Leitura musical vicária. \\ Leitura, comunidades interpretativas e discurso sonoro: o caso do Hei mihi Domine do Francisco Guerrero e sua leitura na Catedral de Bogotá
}

\author{
Miguel Fonseca Martínez
}

\section{Resumo}

$\mathrm{O}$ objetivo fundamental do presente artigo consiste em vincular o conceito de comunidades interpretativas a uma possível hermenêutica da música. Deste modo, a linguagem musical poderia ser interpretada mais facilmente se for descrita analogicamente com sua raiz linguística. Disso devém que uma descrição das praticas e comunidades interpretativas da música estariam amplamente relacionadas com as praticas e comunidades interpretativas das linguagens naturais. Esta tentação metodológica quer razoar através de um caso. Pretende-se ver a plausibilidade da hipótese com um experimento. $\mathrm{O}$ experimento consiste em mostrar como foi lida a música do Francisco Guerrero na Bogotá da Colônia e assinalar se nesse caso existem relações com o desenvolvimento da leitura de linguagens naturais ou se, pelo contrario, há descontinuidades. Quer-se ver se o conceito de comunidades interpretativas pode dar conta, até certo ponto, da dialética entre obra musical 
e o sentido extratextual da linguagem musical. Se tentará mostrar que talvez a música não seja somente uma linguagem sintática, senão que sua semântica pode devir do extramusical que entra nas praticas de quem a escreve e quem a lê.

Palavras-chave: comunidades interpretativas, discurso sonoro, leitura musical vicária, Francisco Guerrero, leitores amadores, leitores profissionais.

"Fuera de la materia, todo es música: Dios mismo no es más que una alucinación sonora”.

—Cioran, Silogismos de la amargura

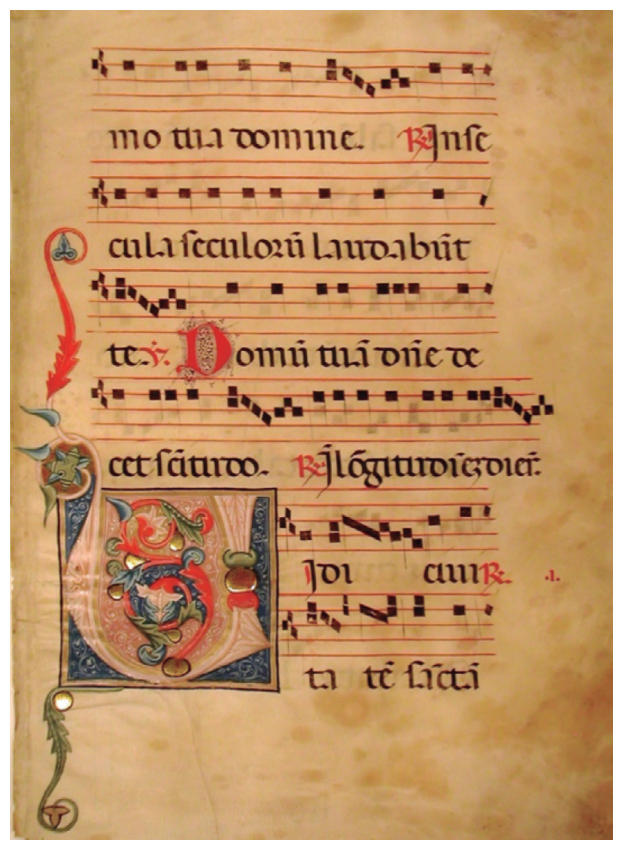

Figura 1. The Eighteen Leaves of A28904 in the Annmary Brown Collection - Folio 16 recto. Brown University Library. 


\title{
Lectura y comunidades interpretativas
}

La lectura no es una agencia que se realice aisladamente y de forma particularizada. El acto de leer se vincula a una "comunidad de interpretación" (Fisch, 1980, p. 167) inherente a un trasfondo histórico. Los actos de habla de cierto periodo temporal, el uso de los significados y los diversos mundos de la vida translucen la manera como los seres humanos construyen ciertos aspectos de la realidad a través de la lectura y, a su vez, constituyen el significado de los textos que leen a partir de agendas sociales que los determinan.

\begin{abstract}
The notion of "interpretative communities", rather than either the text or the reader, that produce meanings and are responsable for the emergence of formal features. Interpretative communities are made up those who share interpretative strategies not for reading but for writing texts, for constituting their properties.
\end{abstract} (Fisch, 1980, p. 14)

El texto no es en sí mismo, siguiendo a Fisch, una entidad que determine con total suficiencia el significado. Este es el lugar donde se hacen patentes las estructuras epistémicas que conforman los pensamientos, deseos, creencias y demás disposiciones de diversas comunidades y, al mismo tiempo, el reconocimiento de dichos significados se desentraña más bien en un sentido pragmático, es decir, no preguntando qué significa el texto, sino cómo tal artefacto afecta y hace cosas con los lectores en otros trasfondos específicos.

In practice, this resulted in the replacing the question- what does this mean? - by another-what does this do? - with "do" equivocating between a reference to the action of the text on a reader and the actions performed by a reader as he negotiates (and, in some sense, actualize) the text. (Fisch, 1980, p. 3)

El modelo de Fisch realiza un cambio en el dominio de la función, ya que el texto no se convierte en el centro del problema de la lectura y la interpretación, sino que lo es el lector, o, mejor dicho, los lectores, sus agendas y su trasfondo.

In my model the reader was freed from the tyranny of the text and given the central role in the production of meaning [...] Objecting to the formalist assumption that the readers job is to extract the meanings that formal patterns 
possess prior to, and independently of, his activities that greatly expanded their scope. (Fisch, 1980, p. 8)

Así, las diversas actividades o prácticas de los lectores constituyen elementos hermenéuticos que desbordan los actos típicos del reconocimiento de una gramática y la decodificación semántica (Fisch, 1980, p. 9). En este sentido, Fisch concibe al lenguaje ordinario y sus correspondientes prácticas como el fundamento para comprender la estructura de la interpretación de textos. La sedimentación de las prácticas cotidianas del mundo de la vida de los productores textuales permitirá asir los caminos adecuados para una interpretación textual auténtica. Afirma al respecto:

My strategy in the essay is to rescue ordinary language from this imporverishing characterization by arguing that at its heart is precisely the realm of values, intentions, and purposes which is often assumed to be the exclusively property of literature. (Fisch, 1980, p. 10)

El reconocimiento del valor de un texto no deviene de algo interno o propio del texto, sino de la decisión de una comunidad que lo erige como testimonio de las creencias a las cuales asienten. Por esto las comunidades de intérpretes surgen a partir de identificar las estrategias y agencias interpretativas que unen e identifican a un grupo de lectores/escritores cuando configuran las propiedades de un texto (Fisch, 198, p. 14). De esto se desprende que "In other words, there is no single way of reading that is correct or natural, only 'ways of reading' that are extensions of community perspectives" (Fisch, 1980, p. 16).

Los procesos de las comunidades interpretativas traen a la vida la obra de arte. En palabras de Wolfgan Iser: "The combined efforts of autor and reader bring into being the concrete and imaginary object which is the work of the mind. Art exist only through other people" (Iser, 1978, p. 108).

Para nuestro caso, es necesario entonces señalar y caracterizar las comunidades interpretativas que permiten el surgir de la obra de arte de musical, el significado de la obra en tanto discurso sonoro. 


\section{Lectura del discurso sonoro: la música como texto}

La escritura y lectura de la música han sufrido un desarrollo concomitante con el desarrollo de la lectura y escritura de los lenguajes naturales. No obstante, su desarrollo posee múltiples discontinuidades que los acercan y alejan en ciertos momentos de la historia. Dicha tarea de establecer una historia de la lectura musical es un mero proyecto que el presente texto quiere empezar a dilucidar con un ejemplo tentativo.

En primer lugar, para poder hablar de una historia de la lectura y escritura musical se debe acotar la definición de la música como discurso sonoro. La música, siguiendo a Eggebrecht, es la estructura formal del sonido, que en ciertas modalidades se actualiza en prácticas concretas en el tiempo (Dahlhaus y Eggebrecht, 2012, p. 188). Sus rasgos esenciales son la mathesis, es decir, el cálculo racional, la emoción y el tiempo. De ello deviene algo análogo a un lenguaje o discurso que tiene como particularidad fundamental la carencia de referentes concretos e incluso de referentes conceptuales definidos; sus referencias son modalidades que se actualizan y concretizan en seres humanos particulares en momentos históricos irrepetibles. La música existe a través de alguien que existe.

Por lo tanto, hay un mero aire de familia cuando se habla de discurso sonoro. Si bien el canto, por ejemplo, usa lenguajes naturales, el sentido de la música que lo contiene no es igual al de un lenguaje natural. Por esto, se puede escribir y leer música, empero la notación es un pretexto para que, al formar el sonido, se exprese un puente para referentes puramente contrafácticos que en absoluto son significados convencionales.

Lo escrito aparece como letra muerta; el espíritu de la obra, se supone, no ha quedado fijado y conservado en la notación, sino que se revela solamente en la comunicación entre el compositor y el intérprete; comunicación que necesita el texto musical solamente como vehículo. (Dahlhaus y Eggebrecht, 2012, p. 191)

El texto musical no es en sí mismo, siguiendo a Fisch, una entidad que determine con total suficiencia el significado. El proceso histórico que permitió fijar en grafías la música, permitió a su vez una doble vía, pues consolidó la autonomía semántica de la obra y a la interpretación como mero medio de expresión de un significado fijo. No obstante, al mismo tiempo, desenvolvió el sentido de musicalidad que desborda lo escrito, como se decía unas pocas líneas arriba. En este sentido, Afirma Dahlhaus: 


\begin{abstract}
Si por un lado parece, pues, que la obra musical —entendida como un conjunto sonoro dotado de sentido- se constituye solo más allá del texto, por otro lado el concepto de obra musical, tal como fue formado paulatinamente entre los siglos XIV y XVIII, incluye la noción de que una composición fijada en notas no es un mero modelo para la práctica musical, sino que viene a ser, por analogía con la poesía, un texto en el sentido enfático de la palabra: una estructura que da forma a una significación expresiva, y cuya representación acústica cumple una función meramente interpretativa [...] Pero en el transcurso de esta evolución se iba divulgando también la convicción de que lo decisivo en la música es lo que no está escrito en las notas. (Dahlhaus y Eggebrecht, 2012, p. 192)
\end{abstract}

De ahí, para solventar dicha aparente contradicción, nace la propuesta de atar el concepto de comunidades interpretativas a una posible historia o hermenéutica de la música. Así, las conexiones de este juego de lenguaje pueden ser dilucidadas más fácilmente si se describen analógicamente con su raíz lingüística, es decir, una descripción de las prácticas y comunidades interpretativas de la música está ampliamente emparentada con las prácticas y comunidades interpretativas de los lenguajes naturales.

Esta pura tentación quiere entonces razonar a través de un caso. Se pretende ver la plausibilidad de la hipótesis con un experimento. El experimento consiste en mostrar cómo se leyó la música de Francisco Guerrero en la Bogotá de la Colonia, y señalar si en este caso existen relaciones con el desarrollo de la lectura de lenguajes naturales o, si por el contrario, hay discontinuidades. Se quiere ver si el concepto de comunidades interpretativas puede dar cuenta en cierta medida de la dialéctica entre obra musical y el sentido extratextual del lenguaje musical. Se intentará mostrar así que quizá la música no sea solamente un lenguaje sintáctico, sino que su semántica puede devenir de lo extramusical que se cuela en las prácticas de quien escribe y quien lee.

\title{
La lectura en la baja Edad Media y el Renacimiento: comunidades interpretativas en tiempos de Francisco Guerrero
}

Para una historia de la lectura, este periodo histórico se comprende como la continuidad del proceso de consolidación del códex como el medio de lectura en Europa desde 
el siglo VII (Saenger, 2011, p. 120). A demás se suma el absolutamente importante cambio lingüístico de la separación de las palabras acaecido en el siglo XII:

Togheter, word separation and word order were conductive to the precise expression of ideas in the clear and unambibiguous manner so essential for communicating the subtle distinctions of scholastic philosophy. They were also prerequisites for the development of syntactic puntuaction and rapid silent reading which depended on the swift visual recognition of Word shape and the perception of larger graphic contours of the page: the clause the sentence and the paragraph. (Saenger, 2011, p. 120)

El cambio que se genera en el denominado Renacimiento de los siglos XII y XIII conllevó a la simetría entre las formas de lectura y cierto tipo de gramática de los textos escritos. La lectura inicia un abandono progresivo de la oralidad y su contingencia, para internarse en el paradigma preponderante de lo visual. Dicha gramática extrañamente no se vincula en un primer momento a las leyes de la lógica o la dialéctica, sino, más bien, a la normatividad de la correcta escritura musical que veremos adelante; las estrategias comunicativas del discurso sonoro, o lenguaje musical, fueron adoptadas por su hermana, la escritura de lenguajes naturales. Al respecto, nos ilustra Saenger:

John of Salisbury, like Hugh of Saint Victor, distinguished in his vocabulary between the reading aloud of a master to a student (praelectio) and private silent, visual reading (lectio). Like the late eleventh century masters of the art lectoría, John viewed grammar as including the art of correct writing, and he regarded puntuaction as paratextual signs for communicating between autor and reader analogous to the neumes used for the notation of music. (Saenger, 2011, p. 123)

La lectura en voz alta, análoga por supuesto a una lectura musical, se sedimenta a usos sobre todo piadosos que permitan el affectus cordis. De otro lado, y dada la nueva cultura visual, aparecen copistas o Pictores que realizan manuscritos, que generalmente no entienden, a los cuales añaden índices bien apuntalados, miniaturas ilustrativas y pedagógicas, al igual que paginación y márgenes. En este sentido, la Escolástica inició el proceso de la composición de manuscritos elaborados por el mismo autor, pues la extensión y complejidad de sus escritos hacía poco plausible acudir a amanuenses sin entrenamiento intelectual. No obstante, las prácticas de los Pictores fueron asumidas 
como costumbre para la práctica de los manuscritos autógrafos que derivarán en un concepto más restringido de autoría. Entre ellas se destaca el uso de la fuente gótica cursiva, que permitía la revisión y recomposición de las ideas que se plasmaban con una mayor facilidad (Saenger, 2011, p. 132).

Llegado el siglo xiv, los escritores y lectores normalizaron la práctica de la lectura silenciosa y visual como la más apropiada para la hermenéutica de los textos escritos que usan lenguajes naturales:

In antiquity and the early Middle Ages, when texts were composed orally, authors expected them to be read aloud. In the fourteenth century, when texts were composed in silent isolation in coursive script, authors expected them to be read silently. (Saenger, 2011, p. 133)

La enseñanza no fue ajena a este paradigma que se asienta principalmente en las primeras universidades y el surgimiento de las bibliotecas modernas y el sistema de prácticas que aún hoy día mantenemos. La lectura en voz alta era un privilegio del Magistro que guiaba la lectura silenciosa de las copias autógrafas de los estudiantes. La lectio divina de los monjes se reservó entonces para los usos piadosos (Saenger, 2011, p. 133).

De esto deviene el aumento exponencial en el número de manuscritos personales glosados que constituyen una nueva comunidad de lectores entre la afición y el profesionalismo. La obtención de conocimiento a través del entrenamiento intelectual permitió el florecimiento de una comunidad de lectores profesionales. La universidad se convirtió en el proveedor de instrumentos intelectuales para interpretar, explicar y comentar los textos (legere), discutir aspectos puntuales (disputare) y la discusión pública de la dimensión espiritual (praedicare).

Esto permitió la formación de una comunidad de nuevos lectores, "groups that shared a programme of intellectual training such as the religious orders" (Hernández, 2015, p. 4). Los lectores profesionales son caracterizados, como se decía, por el modelo del entrenamiento escolástico. Su lectura es preferiblemente personal, privada, silenciosa y usa la biblioteca como una herramienta de trabajo. Afirma el profesor René Hernández al respecto: "More often than not, this reader handles copies of books written 'manu propia' which shows his specialist relationship to writing" (Hernández, 
2015, p. 5). Las glosas o notae bene son la evidencia más clara de este tipo de técnica y práctica de lectura.

El lector aficionado, por el contrario, asume una práctica caracterizada por la lectura en voz alta y en público; no comprende al texto como un instrumento, sino como un objeto simple y, por lo tanto, no se encuentran glosas ni marcas en los manuscritos sujetos a su actividad, excepto las denominadas maniculae o marcas de lugares específicos para retomar la lectura (Hoorebeek, 2014, p. 130).

Tal categorización en este momento de la Baja Edad Media, sin embargo, no es absolutamente categórica, pues las prácticas seguían traslapándose en ciertos contextos restringidos (Hernández, 2015, p. 7). Los dictados, la predicación y la lectura en voz alta, permitían conservar una visión más ortodoxa, institucional y controlada de las disciplinas, empero la lectura visual de la emergente comunidad de lectores permitió el nacimiento de perspectivas heterodoxas y agendas ocultas propias de este momento de encabalgamiento histórico.

Reading with the eyes alone and written composition removed the individual's thoughts from the sanctions of the group, and fostered the milieu in which the new university and lay heresies of the thirteenth centuries developed. (Saenger, 2011, p. 137)

La escritura de los lenguajes naturales adquirió gracias a esta nueva comunidad de lectores la posibilidad de separarse definitivamente de sus orígenes musicales, en el sentido de abandonar una lectura rítmica en función de la oralidad (Saenger, 2011, p. 140). Los nuevos artefactos textuales desencadenaron así la necesidad de la revolución de la imprenta atada a la nueva visión de mundo del protestantismo.

The printing press would play an important role in the ultimate triumph of Protestantism, but the formulation of reformist religious and political ideas and the receptivity of Europe's elite to making private judgments on matters of conscience owed much to a long evolution that began in the late tenth century and culminated in the fifteenth century in the manner in which men read and wrote. (Saenger, 2011, p. 148) 


\section{Escritura y lectura musical en la Baja Edad Media y el Renacimiento: el inicio del concepto de obra}

La escritura y notación musical inician específicamente con la normalización del Canto gregoriano en la Edad Media, y se consolidan como un sistema más o menos estable en el Renacimiento con un apuntalamiento casi definitivo a finales del Barroco. Se pueden observar, sin embargo, desarrollos pioneros respecto a la notación desde la Grecia clásica. El alfabeto del dialecto jónico servía como sistema artificial para referir los tonos de los denominados tetracordios o cuatro tonos que se configuraban o bien diatónicamente, cromáticamente, o enarmónicamente. Esta organización atada al concepto de modos se conserva en cierto sentido en la tipología de escalas contemporánea.

No obstante dichos esfuerzos, la transmisión de la música se realizó principalmente a través de la oralidad. Si bien ya en el siglo vir los textos del canto figuraban en manuscritos, las melodías no se escribían de ninguna manera. La memoria y la representación de los humanos era la única forma de poseer la tradición musical. El texto de los lenguajes naturales y su rítmica, inherente a su convencionalidad y sintaxis, se convertían en una suerte de guía para los acuerdos melódicos que cada comunidad escogía para interpretar su música.

Así, y por esta relación con el texto surgieron los neumas o gestos. Sobre los manuscritos del lenguaje natural se escribían los dichos neumas para indicar el número de notas que se interpretarían, al igual que su posición tonal hacia arriba o hacia abajo (Grout, 2008, p. 134). 


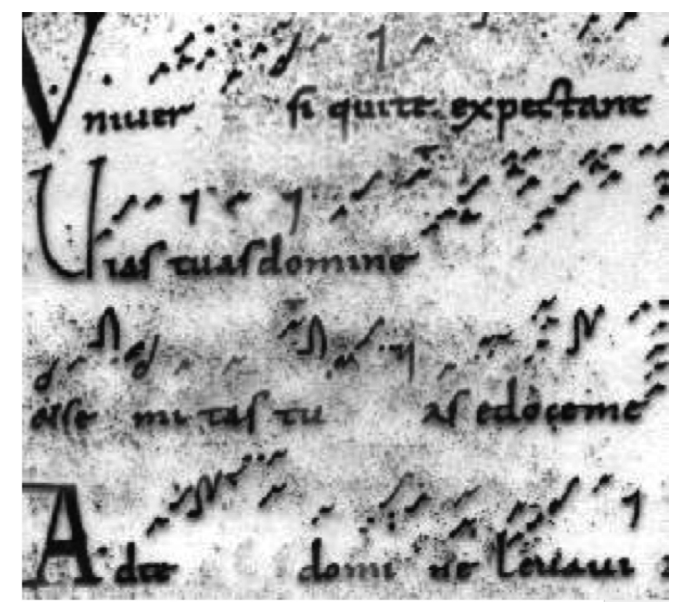

Figura 2. Escritura con neumas. Fragmento de Laon del Ad te Domine levavi pour le premier dimanche de l'Avent, Folio 8.

La revolución respecto a la escritura musical se da en torno a la figura de Guido Mónaco o Guido de Arezzo (992-1050). Guido asumió el uso del alfabeto árabe y el himno de Juan Bautista como pretexto para nombrar las notas musicales y sistematizar, casi que definitivamente, la escritura de la música. En el alfabeto árabe se usaron las letras mim, fá, sad, lam, sin, dal y ra, como guías convencionales para el inicio de frases musicales. Esta contribución de la cultura islámica fue adoptada por Pablo el Diácono, quien compuso el Himno a San Juan Bautista usando dichas sílabas representativas de la escala, como iniciales de sus versos de la siguiente forma:

Ut queant laxis. Resonare fibris. Mira gestorum. Famuli tuorum. Solve polluti. Labii reatum. Sancte Ioannes.

Así, Guido desarrolló el concepto de la delgada línea roja, es decir, un punto de referencia convencional que se refiere a la altura tonal de $f a$, complementado por una línea amarilla en el do, y que derivaría en el tetragrama como sistema de escritura. Guido obvió la séptima, pues al generar un tritono le concedía un carácter diabólico para su tiempo. Ulteriormente este sistema se modificó añadiendo tal tono y constituyendo el pentagrama que hoy día conocemos. La escritura neumática que procedía con el punctus y la virga como barras inclinadas a la izquierda y la derecha que representaban la variación ascendente o descendente de un tono, fueron remplazadas por las notas cuadradas, debido al bisel de las plumas que se usaban para escribir (Palisca, 2001). 


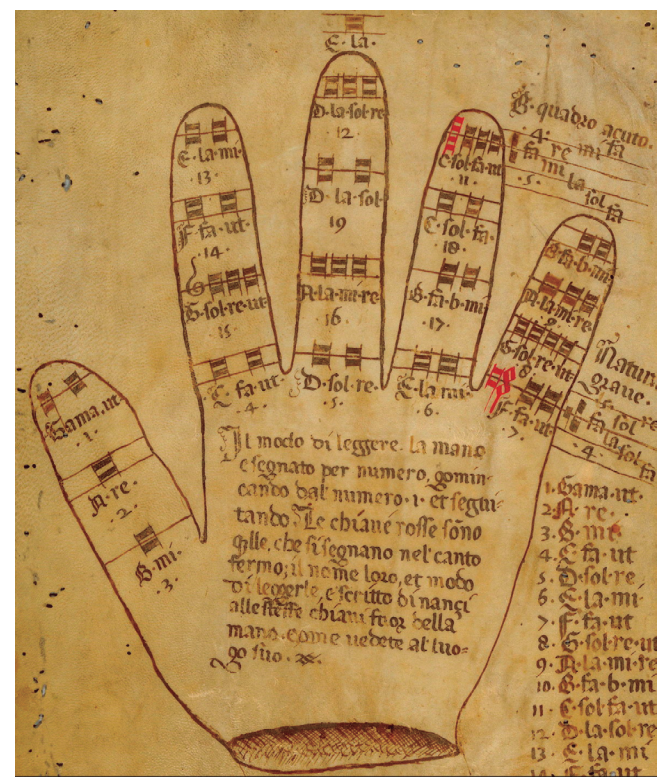

Figura 3. Mano Guidoniana, Oxford University ms Canon.

Liturg. 216. f.168 recto. Bodleian Library.

Respecto a la representación convencional de la duración, la Edad Media usó los modos de Johannes Garlandia que se apoyaban en modelos estandarizados de ligaduras según fuese una nota longa o breve:

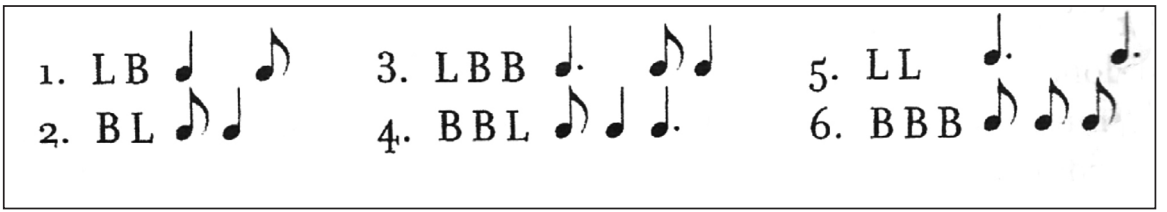

Figura 4. Representación de la notación de Garlandia y su equivalente actual.

Tales seis modos se nombraban también en relación con formas de versos poéticos como troqueo, yambo, dáctilo, anapesto, espondeo y tribaquio. Solo hasta el siglo XIII, con la composición de música principalmente silábica, se vio la necesidad de una escritura convencional más precisa en manos de Franco de Colonia y su tratado Ars cantus mensurabilis: 


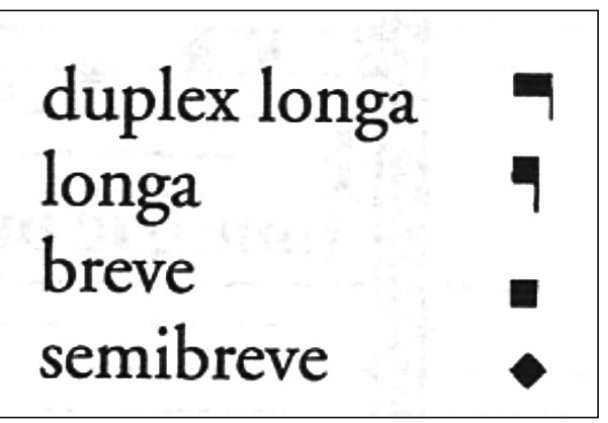

Figura 5. Semántica de la notación mensurada del siglo XIII.

Este tipo de escritura constituyó la normalización de la escritura musical al final de la Edad Media y en el inicio del Renacimiento, con pequeñas modificaciones en las prácticas. En primer lugar, dada la escritura en papel y no en pergamino, las notas solían ser escritas no con un relleno negro, sino huecas, para evitar salpicaduras en el papel. Se añadió el concepto de mínima o blanca actual y sus marcadores con rellenos en la cabeza para hablar de semimínima o negra, y ulteriormente de fusa, que equivale a nuestra corchea.

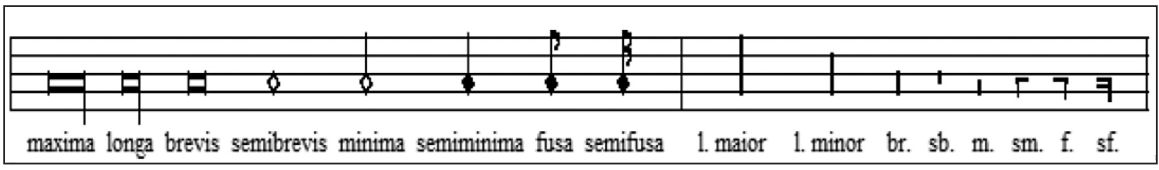

Figura 6. Representación mensurada a comienzos del siglo xv.

Esto llevó a un cambio en la velocidad de las composiciones y permitió el virtuosismo como un nuevo ingrediente técnico en la interpretación. Gracias a estas novedades, se logró una mejor comunicación de la música polifónica, dada la posibilidad de representar simultáneamente sonidos en el papel, y de ello, el aumento de las composiciones contrapuntísticas y el inicio de una fuerte abstracción de la armonía como elemento musical de primer orden. Se sumó a todo esto la aparición de tablaturas o cifras, sobre todo para instrumentos de plectro y órganos. Las cifras estaban dedicadas a los lectores aficionados, pues simulaban las posiciones reales y no convencionales de las notas, por ejemplo, en las cuerdas de la vihuela (Fonseca, 2012). 


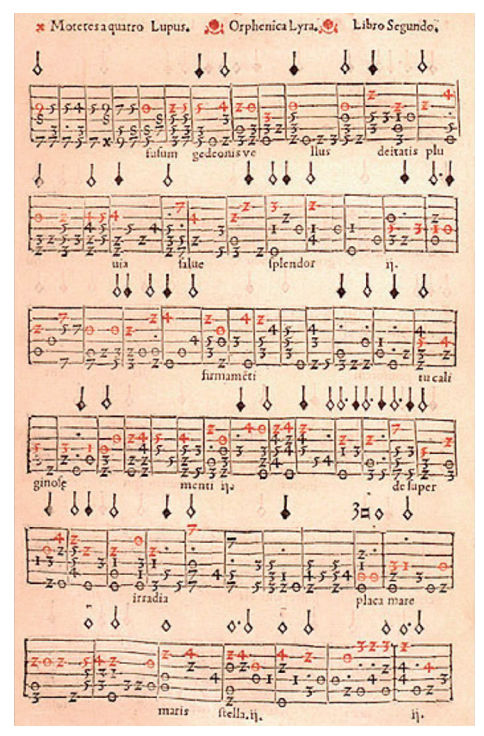

Figura 7. Página del libro de cifra de Fuenellana Orphenica Lyra, 1554.

Todas estas innovaciones se dieron gracias un nuevo pensamiento humanista, el cambio en las ideas del protestantismo y sobre todo a la empoderada casta de los burgueses. La imprenta permitió a su vez el inició de cierta universalización de la música como una forma de suplir la demanda de entretenimiento a las nuevas castas sociales.

La música se objetiva, por lo tanto, como una cosa o producto; se constituye en obra. Las iglesias se convierten así en los principales centros de enseñanza de las técnicas y métodos de interpretación de estos textos cerrados y con sentido propio. Tal enseñanza deriva en una lectura profesional que comprende perfectamente la gramática del sistema artificial o convencional, y de otro lado, una lectura aficionada o diletante que muestra o traduce los sistemas a los menos ilustrados. De ello surge la casta de los cantores o maestros de capilla, que generalmente eran clérigos que se dedicaban a una lectura profesional de la música e iniciaron una comunidad de lectores especializada que se difundió prontamente por Europa y ulteriormente en sus colonias. El pueblo se aproximaba vicariamente a la lectura de la música. En el transcurso del Renacimiento se presentó una secularización fuerte que constituyó a un grupo de lectores profesionales de la música en una suerte de gremio de artesanos pagados que perduró con esta configuración hasta finales del Barroco. 


\section{La obra de Francisco Guerrero}

Francisco Guerrero (1528-1599) nace en Sevilla e inicia sus estudios musicales en familia con su hermano Pedro, a quien alaba en su autobiografía de 1593 Viage de Hierusalem (Guerrero, 1593) por su conocimiento y estricta enseñanza. Cantor, desde la niñez en la catedral de Sevilla, se caracterizó por su extraña voz de contralto. Tras la ausencia de su hermano, usa la doctrina musical de Cristóbal de Morales para sus composiciones, de la cual tiene testimonio directo en su visita a Toledo hacia 1545. Por la bula Pastoralis officii de Julio III de 1554, y tras diversos devaneos entre la maestría de Jaen y Málaga, fue nombrado a perpetuidad maestro de capilla de la iglesia de Sevilla (Llorens, 2000, p. 26).

Se destacan entre sus principales presentaciones las audiciones ante Carlos I gran conocedor y mecenas de la música. Se destaca la anécdota, según la cual, el emperador mismo, al revisar un libro con misas y motetes que Guerrero le obsequió, acusó a Guerrero de plagio. Francisco se defendió vehementemente hablando de la práctica de las misas parodias como pretexto para la composición improvisando voces, es decir, la composición de motetes. Su discurso fue más que suficiente para lograr la solicitud de excusas de parte del emperador y su exaltación como compositor (Llorens, 2000, p. 26). Así, por esta bien lograda fama, en su audición con Felipe II en Madrid en 1561 le fue permitido ser parte del séquito de músicos del matrimonio del monarca con la princesa Ana de Austria (Llorens, 2000, pp. 27-28).

El prestigio ganado le permitió viajar a Roma y Venecia, centros editoriales de renombre, publicando el Liber secundum missarum en 1582. El texto ofrecido en audiencia particular al pontífice Gregorio XIII fue aprobado no solamente por este, sino por los colegas que allí encontró, incluyendo al gran Tomás Luis de Victoria.

A sus sesenta años de edad logró tras un mes de navegación su sueño de niñez de conocer tierra santa. El viaje narrado en su Viage de Hierusalem (Guerrero, 1593) simula la estructura de Dante y su entelequia es principalmente piadosa.

A su regreso se encarga de las tareas de enseñanza, administración y composición en su puesto vitalicio. Por querer publicar sus motetes en Venecia en 1589, se endeudó ampliamente, de tal suerte que, al no poder pagar, fue encarcelado en Sevilla hasta que su deber fue pagado por la municipalidad en recompensa a sus servicios y por su avanzadísima edad para su tiempo y el nuestro (Llorens, 2000, p. 27). 
Francisco Pacheco, su retratista y suegro de Velázquez, destaca en las glosas del retrato sus calidades humanas y artísticas, y su práctica vital casi piadosa.

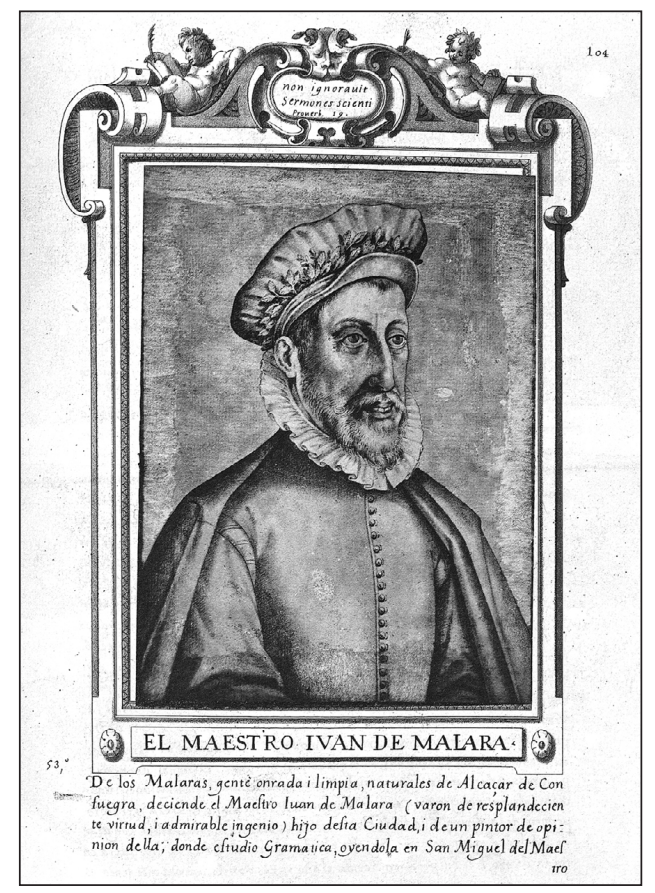

Figura 8. Retrato tomado de Francisco Pacheco (1564-1644), El libro de descripción de verdaderos retratos, ilustres y memorables varones [Sevilla, s.n., s.a.]. Real Academia de la Historia (Madrid). Signatura: 1/736.

Góngora, en su Romance 35, resume maravillosamente el estilo de Guerrero: "Cuanto porque el español/en las lides que le mete/hace más fugas con él/que Guerrero en un motete" (Góngora, 1999).

La esencia de las obras de Guerrero consiste sobre todo en la imitación libre de cantus firmus a través de estructuras polifónicas a contrapunto, con abundantes cánones y fugas. Para ello se valió de parodias de otras obras e incluso de canciones populares. Afirma al respecto Llorens, editor de la Opera Omnia, refiriéndose a la técnica contrapuntística: 
El discurso melódico procede generalmente por grados conjuntos, en tanto que los intervalos disjuntos de tercera, cuarta y quinta prevalecen a los de sexta y octava. El ritmo está regulado por los acentos tónico-expresivos, simultáneamente ordenados hacia una cantilación media. La combinación del ritmo ternario con el binario corresponde al principio agustiniano de hacer depender la música del texto penetrando en la entraña semántica de una palabra o frase, particularmente evidenciable en el repertorio de cantos humanos, algunos de ellos "convertidos a lo divino". La búsqueda de la unidad formal a través de la integración de las partes es otra constante coherente con el pensamiento de San Agustín, filósofo; así como el valor de los símbolos que se descubre a través del minucioso de su velado y sutil ingenio. En la forma estructural se distingue por el modo de tratar proporcionalmente cada una de las frases de los textos literarios. (Llorens, 2000, p. 33)

La obra del gran músico hispalense es recogida en la denominada Opera Omnia (Guerrero, 1986). Las fuentes manuscritas que se presentan en el Liber secundum, es decir el volumen 5 de la recopilación del Instituto Español de Musicología, se encuentran en los archivos de Toledo, Sevilla y Viena, al igual que en la edición impresa de Roma de 1582. En el archivo de Sevilla se encuentran algunas de las misas en pergaminos de 530 X $365 \mathrm{~mm} 46$ ff, que, por ejemplo, en la numeración 1-19 se refieren a la misa Iste Sanctus. Todos estos folios fueron encuadernados treinta y ocho años después de la muerte de Guerrero en 1599 y adornados por Andrés Camacho con cifras romanas de color rojo, iniciales grandes y adornadas; encuadernación con tapas de madera cubiertas de piel y clavos decorativos para el culto de la Capilla de Nuestra Señora de la Antigua (Llorens, 1986, p. 9). Este manuscrito, que se refiere al Liber secundum missarum, es algo así como un segundo manuscrito mejorado, o bien de los manuscritos originales, o bien de la obra impresa en Roma que se encuentra en el archivo de Toledo. En este mismo sentido, el códice de Viena Ms 897 de la Österreiche Nationalbibliotek se considera una réplica de la lujosísima edición impresa Missarum liber secundus editada en 1582 en Roma y que precede cronológicamente a los manuscritos. Los denominados pautados y semitonos de las obras coinciden en ambos textos. No obstante, la acentuación de las palabras difiere, no se corresponden perfectamente la duración de las notas y existen palabras que se iteran más en uno que en otro. Además, en el folio 1 se quiso modificar el nombre de Guerrero por el de Palestrina, considerando a Guerrero un copista más de su 
música (Llorens, 1986, pp. 11-12). El caso es igualmente patente en el archivo de Toledo (Llorens, 1986, p. 10).

Se puede inferir de lo antedicho que la autoridad sobre las obras ha sido dictada por los textos impresos, y que los manuscritos son diversos códices sin unidad de criterios que muestran la práctica muy idiosincrática de los copistas de la época de alterar el contenido de las obras, para ciertos propósitos particulares. El concepto de autoría aún estaba en formación, y por ello, como se refería más arriba, parodiar obras era una costumbre muy popular. De otro lado, los manuscritos se constituían, como se afirmaba en el anterior acápite, en herramientas de aprendizaje que iniciaban una incipiente profesionalización en la lectura musical. Los textos impresos — valga decir, los primeros textos impresos-, seguían representando al texto como un objeto y no tanto como una herramienta. Por ello, los manuscritos seguían siendo un elemento o artefacto más adecuado para el uso que para la contemplación. No obstante, en tanto textos fijados, los libros publicados constituían el cierre ontológico del texto musical en tanto obra y, de ello, la propiedad de la autoría y su significado también como un concepto cerrado.

Así, la fuente principal del denominado Liber secundus es el cancionero de Roma de 1582 que incluye algunos motetes adicionales a las cuatro misas patentes en este y los códices.

El Missarum liber secundus Francisco Guerreri in alma Ecclesia Hispalensi Portionarii, et cantorum Praefecti (Roma, 1582), nombre original de la edición realizada por F. Zanetti, es, pues, el testimonio más fiel de la obra de Guerrero. De este se conservan 28 copias, en 19 ciudades, entre ellas, 8 en Italia, 9 en España, 1 en el conservatorio de París y la última en la Catedral de Bogotá (CO-B). Escrito en Latín, contiene 9 obras que incluyen 8 misas y un motete. Estos son, a saber:

Op 115 Hei mihi Domine; Op 172 Missa Batalla Escutez; Op 200 Missa De Beata Virgine; Op 214 Missa Ecce Sacerdos; Op 235 Missa Iste Sanctus; Op 269 Missa Pro Defunctis; Op 283 Missa Puer qui natus; Op 304 Missa Simile est regnum caelorum; Op 318 Missa Surge Propera (Guerrero, 1582). 


\section{El Missarum liber secundus en el archivo musical de la Catedral de Bogotá}

El archivo fue inventariado y catalogado por vez primera en 1961 por Robert Stevenson (Cabo, 2012, p. 4). Ulteriormente José Ignacio Perdomo publicó una investigación relativa a los villancicos que se encontraban en el archivo en el año de 1973 titulada El Archivo Musical de la Catedral de Bogotá (Perdomo, 1973). Tales trabajos eran principalmente descriptivos y se explicitaban en inventarios, transcripciones y breves historias de la música colonial. Un siguiente estudio sobre el cerrado archivo eclesial sugirió una visión más descriptiva en las obras de Egberto Bermúdez y Elie Ann Duque (1982-1985-1996). Sus trabajos apuntaban a una historia social de la música y a un esclarecimiento del trabajo mismo de la musicología bajo el pretexto de un estudio de las obras del archivo. El archivo no ha sido un espacio plenamente abierto y los estudios sobre este han sufrido cierto sesgo eurocéntrico y positivista atado a los contextos socioculturales a los cuales han pertenecido los pocos estudiosos de este (Cabo, 2012, p. 7).

La historia del archivo puede fijar su origen en 1550 con el arribo del arzobispo Juan de Barrios, primer arzobispo de Santafé de Bogotá. El canónigo que trazó las basas de la construcción de la Catedral trajo consigo algunos libros de canto llano para las actividades canónigas (Zamora, 1962).

El siguiente hito lo constituye la llegada del mestizo Gonzalo García Zorro desde Sevilla con la suma del libro de magnificats Canticum Beatissimae Virginis Deiparae Mariae de Sebastián Aguilera de Heredia y el Missarum liber secundus de Francisco Guerrero (Cabo, 2012, p. 11). 


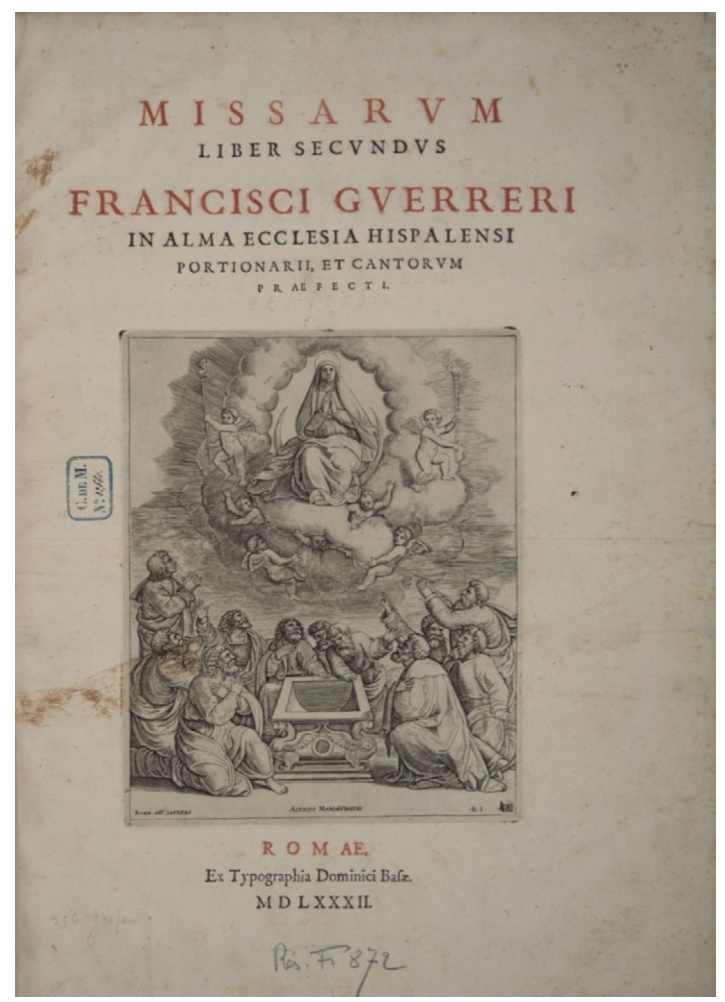

Figura 9. Portada del Missarum liber secundus. In alma Ecclesia Hispalensi Portionarii, et cantorum Praefecti. Roma 1582.

Gonzalo García Zorro fue hijo del capitán Gonzalo García Zorro y de la india Margarita, sobrina de Tisquesusa. Este, sobrino de Nemequene y sucesor del zipazgo, era el líder de los habitantes de la sabana a la llegada de los adelantados (Fernández, 1688, pp. 96-98). García Zorro llegó a convertirse en el primer canónigo mestizo de América y primer maestro de capilla mestizo de Santafé entre 1573 y 1584. El viaje que tenía como pretexto la búsqueda de su legítimo nombramiento, dada su situación de mestizo, permitió el acto fundacional del archivo con una de sus joyas más preciadas, es decir, la obra de Guerrero.

El maestro de capilla Alonso Garzón de Teuste, también mestizo procedente del Cauca, consolidó esta primera etapa del archivo. Ello gracias a la sofisticación en 
la concepción del contrapunto del maestro de Timaná y el encargo del arzobispo Bartolomé Lobo Guerrero de la copia y decoración de 32 libros de canto llano a Francisco Páramo. Los folios contienen letras capitales, viñetas, orlas y miniaturas firmadas por Paramo y glosadas por Tehuste para hacer un uso adecuado de estas (Cabo, 2012, p. 11). En el año de 1613 llega una remesa de veintidós cajas de libros de canto llano desde el monasterio del Escorial a través de la casa de Contratación de Sevilla, principalmente con obras de Guerrero, Cristóbal de Morales y Tomás Luis de Victoria (Bermúdez, 1996, p. 46).

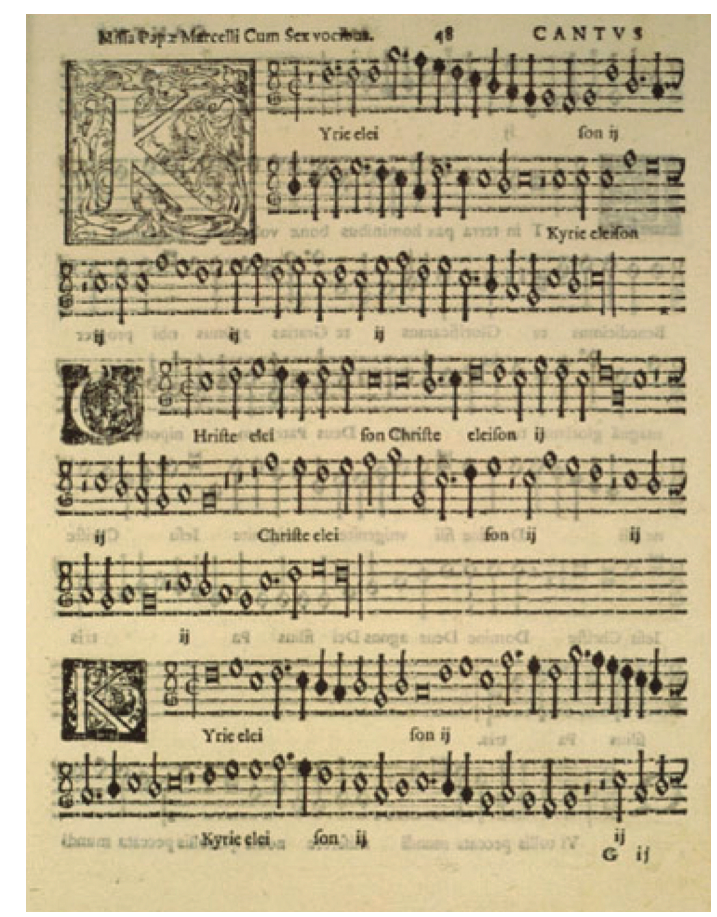

Figura 10. Página del Missarum liber secundus. In alma Ecclesia Hispalensi Portionarii, et cantorum Praefecti. Roma 1582. Kyrie eleison Op 269 Missa Pro Defunctis.

Las guerras de Independencia marcaron el declive de la actividad musical en la catedral y el deterioro del archivo. Según Perdomo, hacia 1860 todo se resumía a ocho bultos de folios calificados en su momento de inútiles (Perdomo, 1976, p. 16). 
El denominado Bogotazo contribuyó a dicho detrimento, pues sirvió de refugio a muchos que en absoluto comprendieron el significado de los folios usándolo para los peores menesteres (Perdomo, 1976, p. 15). Así, como se mencionó arriba, solamente hasta la segunda mitad del siglo xx se rescató gracias a hombres como Perdomo y Stevenson el valor del fondo de la Catedral que, en comparación con los de otras ciudades latinoamericanas, si bien no por su extensión, tiene quizá los mejores testimonios de la música del siglo xvi en América.

\section{El caso del Hei mihi Domine de Francisco Guerrero y su lectura en la Catedral de Bogotá}

Los motetes, cuyo nombre encuentra su origen en el francés mot, es decir, 'palabra', son cierta forma de composición polifónica con origen en la Edad Media, que tenía como propósito ser cantada en las iglesias, sin ser parte de la liturgia. Los textos de los motetes son principalmente bíblicos, mas no litúrgicos, y en latín. Su carácter es antifonal, es decir, un canto responsorial. Dado lo costoso de los pergaminos, los motetes dejaron de ser escritos en ciertos momentos como partituras, a la manera de un score contemporáneo, y se convirtieron en pequeños libros de coro para los cantantes y la congregación. Se fundaban en una melodía dada por el mismo texto o cantus firmus, que para el caso específico de los motetes es denominada ténor, a la que en forma de discanto - o sea, la suma de voces paralelas- se improvisaba la polifonía. Dicho duplum, o añadido de palabras, es el origen de la denominación motete o palabra. Cada voz se refiere entonces a un texto diferente. La mayoría de motetes tenían como rasgo distintivo el denominado hoquetus, oquedad o eco al final de las composiciones, que consiste en la alternancia rítmica de responder la misma nota en el silencio ulterior a la primera y a contratiempo (Hoppin, 2002).

El Hei mihi Domine es un motete que se cuenta como obra independiente. No obstante, hace parte de la misa Pro defunctis que aparece en la misma edición. Se encuentra, pues, entre el Benedictus y el Agnus Dei. Se cantaba antifonalmente en el momento de la elevación. Esta es la estructura del texto: 


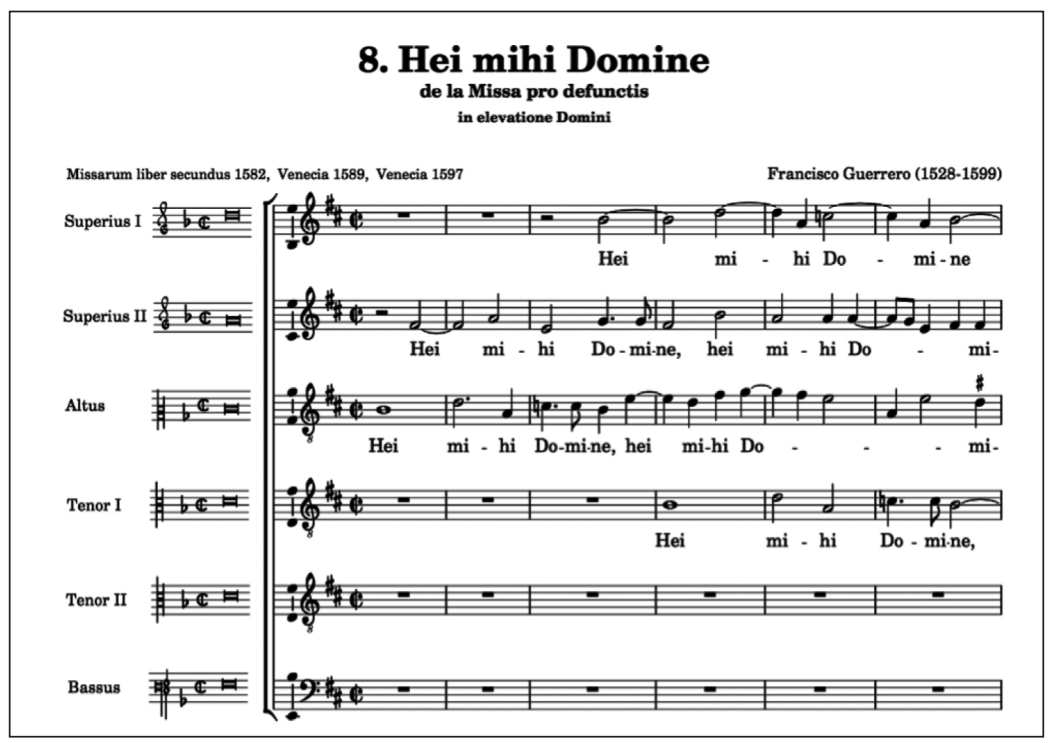

Figura 11. Primera página de la transcripción del Hei mihi Domine Opera Omnia Francisco Guerrero.

Hei mihi, Domine, quia peccavi nimis in vita mea:

quid faciam miser, ubi fugiam, nisi ad te, Deus meus?

Responsum

Miserere mei, dum veneris

in novissimo die.

El texto que se constituye en una súplica y un acto de contrición por la condición del pecado, implora la misericordia en el final de los días. Es el momento cumbre en la celebración de un Requiem. La composición de Guerrero involucra al público en la celebración, dado el carácter antifonal antes descrito, y por ello, les lleva a una lectura musical vicaria. La obra es una forma de compartir musicalmente la muerte.

La misa por los difuntos de Guerrero era una de las obras más interpretadas en el oficio de los maestros de capilla de Bogotá, y este momento, quizá, uno de los pocos donde se involucraba a la comunidad como parte de la interpretación musical. El aprestamiento musical de las colonias no implicaba en esos días (y quizá aún en los 
nuestros) siquiera una lectura aficionada. Solamente en la imitación de las voces de los cantos se comprendía la música, pues incluso difícilmente se lograría una comprensión del texto en lenguaje natural de forma directa. De esto se supone que los maestros de capilla se reservaron la lectura profesional de la música y constituyeron una lectura vicaria o imitativa para sus músicos y las comunidades que pastoreaban.

El texto del Missarum liber secundus del archivo de Bogotá muestra muy poca manipulación, lo cual señala que los mismos maestros entendían el texto musical como un objeto sagrado y se dedicaban a realizar copias manuscritas aún más adornadas donde difundían y quizá enseñaban las obras, pero con muy pocas glosas y comentarios. Es extraño el tratamiento de un texto impreso con tanta devoción, pero se explica por el significado de la obra, es decir, de una edición musical mucho más fiel a lo que Guerrero quiso decir.

La interpretación de la obra, no obstante, debió derivar en una práctica arcaica de memoria e imitación, pues no se contaba con una alfabetización musical suficiente de parte de la comunidad que se quedaría sumida en una fuerte involución musical, más allá de haber bebido prontamente de los mejores exponentes de la música universal de su tiempo.

Se debe notar, en este sentido, que este carácter dialogal evidente en esta miniatura musical será una de las fuertes influencias de Guerrero en la música sagrada protestante y, sobre todo, en el concepto de cantata de Bach. La congregación es parte de la performación y del sentido de la obra musical. En estos tipos de composiciones se realiza una lectura en voz alta, una conducción del sentido de lo sagrado guiada por un maestro con miras a la evangelización. Un ejemplo muy cercano es la canta 179 de Bach (Pirro, 2014).

Esta dinámica de lectura se transformará precisamente en el mismo Bach y su descubrimiento de la música pura en obras como el Arte de la fuga, que atado a cambios políticos transformará al músico de artesano profesional en un artista o incluso un académico del discurso sonoro en el trasfondo de la Revolución Francesa y el Conservatorio Musical.

Sin embargo, en el ámbito latinoamericano esta idea será mucho más tardía y preconizará la lectura musical como una mera imitación basada en textos del lenguaje natural y la autoridad de los maestros de capilla como los principales alfabetizadores de la música. 
La música del Hei mihi Domine interpretada en los albores de la Colonia en Bogotá es, pues, una forma de discurso funcional o subordinado a la argumentación del lenguaje natural por las consecuencias negativas que quiere apelar al sentimiento de una comunidad y su percepción de la contingencia de la vida y la necesidad de la idea de la trascendencia encarnada en la Iglesia católica.

\section{La lectura musical vicaria en la Bogotá del siglo xvI}

El Hei mihi Domine es, como se decía, un caso de la lectura musical vicaria que quizá aún hoy día perdura en las comunidades lectoras musicales de Colombia. Permanecemos, pues, en un paradigma medieval de la lectura musical. La lectura musical era, jugando con las palabras de Schopenhauer, un ejercicio que se hacía inconscientemente mientras se estaba ejecutando. Las comunidades lectoras configuraban el sentido del texto heteronomamente, y mediado por la autoridad del maestro de capilla. Si bien el artefacto textual, esto es, las partituras y los textos, lograron la sofisticación que se describió arriba, no coincidía dicha bondad del artefacto con su uso y práctica. Es decir, las ventajas de la escritura musical de la baja Edad Media y el Renacimiento no se correspondían con su ejercicio epistémico. La música seguía siendo, como hasta el siglo viI, una mera variación incomprensible del lenguaje natural, sin autonomía semiótica y mucho menos semántica.

La posible comunicación de los textos y del discurso sonoro a través de impresos no fue el caso y se retornó extrañamente a manuscritos usados de forma aficionada por parte de lectores profesionales, que no permitieron la construcción de comunidades amplias de lectores profesionales del discurso sonoro y mucho menos de comunidades de lectores aficionados. Esto se vino a dar muy tenuemente hasta finales del siglo xix e inicios del xx. 


\section{Referencias}

Bermúdez, E. y Ann, D. E. (2000). Historia de la música en Santafé y Bogotá. Bogotá: Fundación de Música.

Cabo, L. (2012). El archivo musical de la Catedral de Bogotá: Estudios musicológicos 1938-2009 (Trabajo de grado de Máster). Madrid: Universidad Complutense.

Dahlhaus, C. y Eggebrecht, M. (2012). ¿Qué es la música? Barcelona: Acantilado.

Fernández Piedrahita, L. (1688).

Fisch, L. (1980). Is there a text in this class? Cambridge: Harvard University Press.

Fonseca, Miguel. (2012). Filosofía de la música. Bogotá: Editorial Universidad de La Salle.

Góngora, L. (1999). Romances. Barcelona: Quaderns Crema.

Grout, D. J., Burkholder, J. P., Palisca, C. V. (2008). Historia de la música occidental. Traducción de Gabriel Menéndez Torrellas (séptima ed.). Madrid: Alianza.

Guerrero, F. (1593) (1986). Opera Omnia. Vol. V. Missarum liber secundus. Barcelona: Consejo Superior de Investigaciones Científicas.

Hernández, V. R. (2015). Amateurs or professionals? Reformed Observant Franciscan Friars as Readers. Leeds: International Medieval Congress Session 632. Julio 72015.

Hoppin, R. (2002). Antología de la Música Medieval. Madrid: Akal.

Iser, W. (1999). The Act of Reading. Baltimore: The Jhons Hopkins University Press.

Llorens, J. (1986). Opera Omnia. Vol. V. Estudio Intriductorio al Missarum liber secundus. Barcelona: Consejo Superior de Investigaciones Científicas.

Palisca, C. (2001). Guido of Arezzo. New Grove Dictionary of music and Musicians. Ed. Stanley Sadie. Macmillan.

Perdomo, J. I. (1976-1980). El archivo musical de la Catedral de Bogotá. Bogotá: Instituto Caro y Cuervo. 
Pirro, A. (2014). The Aesthetic of Johann Sebastian Bach. New York: Rowman and Littlefield.

Rodríguez, C. (2005). Nietzsche contra Wagner: una diatriba ante el romanticismo musical. Cuadernos de Filosofía Latinoamericana, 108-120.

Saenger, P. (2011). Reading in the Middle Ages. In: Cavallo, G. y Chartier, R. (Ed.), A History of Reading in the West. London: Routledge.

Zamora de, Fr. A. (1962). Historia de la Provincia de San Antonio del Nuevo Reino de Granada. Bogotá: Biblioteca de Cultura Colombiana. 